\title{
Microwave-assisted one pot three-component synthesis of some novel pyrazole scaffolds as potent anticancer agents
}

\author{
Sobhi M. Gomha1*(D, Mastoura M. Edrees ${ }^{2,3}$, Rasha A. M. Faty ${ }^{1}$, Zeinab A. Muhammad² and Yahia N. Mabkhot ${ }^{4}$
}

\begin{abstract}
Background: Pyrazoles, thiazoles and 1,3,4-thiadiazoles have been reported to possess various pharmacological activities.

Results: An efficient and a novel approach for the synthesis of some novel pyrazole based-azoles are described via multi-component reaction under controlled microwave heating conditions. The structures of the synthesized compounds were assigned on the basis of elemental analysis, IR, ${ }^{1} H$ NMR and mass spectral data. All the synthesized compounds were tested for in vitro activities against two antitumor cell lines, human lung cancer and human hepatocellular carcinoma compared with the employed standard antitumor drug (cisplatin).

Conclusions: All the newly synthesized compounds were evaluated for their anticancer activity against human lung cancer and human hepatocellular carcinoma cell lines using MTT assay. The results obtained exploring the high potency of six of the tested compounds compared with cisplatin.
\end{abstract}

Keywords: Acetylpyrazoles, Enaminones, Hydrazonoyl chlorides, Thiazoles, Thiadiazoles, Anticancer activity

\section{Background}

Multi-component reactions (MCR) are one-pot processes with at least three components to form a single product, which incorporates most or even all of the starting materials [1-6]. The huge interest for such multi-component reactions during the last years has been oriented towards developing combinatorial chemistry procedures, because of their high efficiency and convenience of these reactions in comparison with multistage procedures. Also, the utility of MCR under microwave irradiation in synthesis of heterocyclic compounds enhanced the reaction rates and improve the regioselectivity [7-12].

On the other hand, pyrazole and its derivatives have drawn considerable attention of the researchers in the past few decades owing to their high therapeutic values. Some of the drugs, possessing pyrazole as basic moiety, like celecoxib [13], deracoxib [14], etoricoxib and atorivodine [15] are already booming in the market. Pyrazole derivatives possess an extensive range of pharmacological activities such as antiinflammatory, antipyretic, analgesic, antimicrobial, sodium channel blocker, antitubercular, antiviral, antihypertensive, antiglaucoma, antioxidant, antidepressant, anxiolytic, neuroprotective and antidiabetic activity [16-23]. Furthermore, pyrazole prodrugs have also been reported to possess significant anticancer activities [24-30]. Keeping this in mind, and in continuation of our previous work on the synthesis of new anticancer agents [31-40], we herein present an efficient regioselective synthesis of novel 4-heteroaryl-pyrazoles, which have not been reported hitherto in a multicomponent synthesis under microwave irradiation and to assess their anticarcinogenic effects against hepatocellular carcinoma (HepG-2) and human lung cancer (A-549) cell lines.

\footnotetext{
${ }^{*}$ Correspondence: s.m.gomha@gmail.com

1 Department of Chemistry, Faculty of Science, Cairo University,

Giza 12613, Egypt

Full list of author information is available at the end of the article
} 


\section{Results and discussion Chemistry}

Multi-component reaction of acetyl pyrazole 1 [41], dimethylformamide dimethylacetal (DMF-DMA) 2 and nitrileimine 4a-d (generated in situ from $3 \mathbf{a}-\mathbf{d}$ with triethylamine) in toluene under conventional heating for $10-15 \mathrm{~h}$ or under microwave irradiation at $150{ }^{\circ} \mathrm{C}$ for 4-10 min. afforded compound $\mathbf{6 a}-\mathbf{d}$ rather than its isomeric structure 8a-d in $66-70$ and $84-90 \%$, respectively (Scheme 1; Table 1). The structure of $\mathbf{6 a}-\mathbf{d}$ was confirmed by their spectral data (IR, MS and ${ }^{1} \mathrm{H}-\mathrm{NMR}$ ) and elemental analyses. For example, the IR spectra of products 6 revealed in each case two absorption bands in the regions $v 1638-1676$ and $1682-1724 \mathrm{~cm}^{-1}$ due to the two carbonyl groups. The ${ }^{1}$ HNMR spectra showed, in addition to the expected signals for the aromatic protons, three singlet signals at $\delta \sim 2.34,2.55$ and 8.92 reveled to the two methyl groups and the pyrazole- $\mathrm{H} 5$, respectively. The mass spectra of products $\mathbf{6 a}-\mathbf{d}$ revealed a molecular ion peak for each one which is consistent with the respective molecular weight. These data are much closer to those reported in literature on similar work [42-44].

Compound $\mathbf{6 a}$ was alternatively synthesized by reacting enaminone 9 (prepared separately via condensation of acetyl pyrazole 1 with DMF-DMF) with 2-oxo- $N$-phenylpropanehydrazonoyl chloride (3a) in toluene containing catalytic amount of TEA under MWI. The obtained product was found to be identical with $\mathbf{6 a}$ in all respects (TLC, mp and IR spectrum) which affords further evidence to all structures $\mathbf{6 a}-\mathbf{d}$. The latter products were assumed to be formed via initial 1,3-dipolar cycloaddition of the nitrileimines $\mathbf{4 a}-\mathbf{d}$ to the activated double bond in enaminone 9 to afford the non-isolable cycloadducts $\mathbf{5}$ which underwent loss of dimethylamine yielding the final pyrazole derivatives $\mathbf{6 a}-\mathbf{d}$.

The results obtained Table 1 indicate that, unlike classical heating, microwave irradiation results in higher yields and shorter reaction times for all the carried reactions. Microwave irradiation facilitates the polarization of the molecules under irradiation causing rapid reaction to occur. This is consistent with the reaction mechanism, which involves a polar transition state [45].

By the same way reaction of acetyl pyrazole 1 with nitrile-oxide 11a, b (derived from reaction of hydroximoyl chloride 10a, b with TEA) and DMF-DMA in toluene under microwave irradiation at $150{ }^{\circ} \mathrm{C}$ gave isoxazoles 13a, b (Scheme 2; Table 1). The ${ }^{1} \mathrm{H}$ NMR spectrum of the product revealed a singlet signal at $9.67 \mathrm{ppm}$ assigned for isoxazole- $5 \mathrm{H}$ proton not isoxazole- $4 \mathrm{H}$ proton $[42-44,46]$ which consistent with the isomeric structure 13 rather than the isomeric structure 15. Moreover, the mass spectrum of $\mathbf{1 3 a}$ and $\mathbf{1 3 b}$ revealed a molecular ion peaks at $\mathrm{m} / \mathrm{z}=506$ and 446 , respectively, which is consistent with their molecular weights.

Furthermore, alternative synthesis of compound 13a was achieved via reaction enaminone 9 with $N$-hydroxy2-naphthimidoyl chloride (10a) under the same reaction condition to yield authentic product 13a (Scheme 2).

Next, our study was extended to investigate the reactivity of compound $\mathbf{1}$ towards thiosemicarbazide and various hydrazonoyl halides aiming to synthesize new pyrazole based-1,3-thiazoles and 1,3,4-thiadiazoles. Thus, acetyl pyrrole $\mathbf{1}$, thiosemicarbazide 2 and $\alpha$-keto hydrazonoyl halides $3 \mathbf{a}, \mathbf{b}$, e were allowed to react in a one-pot threecomponent reaction in dioxane containing catalytic amount of TEA under MWI to afford the arylazothiazole derivatives 18a-c, respectively (Scheme 3 ; Table 1 ). The reaction goes in parallel to literature [32, 35-37].

The structure of the products $18 \mathbf{a}-\mathbf{c}$ was assigned based on the spectral data and elemental analyses. For example mass spectrum of compound 18a revealed molecular ion peak at $\mathrm{m} / \mathrm{z} 542$ and its ${ }^{1} \mathrm{H}$ NMR spectrum exhibited four characteristic singlet signals at 2.32, 2.36, 2.48 and 10.47 assignable to three $\mathrm{CH}_{3}$ groups and $\mathrm{NH}$ protons, respectively, in addition to an aromatic multiplet in the region $6.99-7.93 \mathrm{ppm}$ equivalent to 12 protons. Its IR spectra showed one $\mathrm{NH}$ group band at $3396 \mathrm{~cm}^{-1}$.

The structure of products $\mathbf{1 8}$ was further confirmed by an alternative method. Thus, reaction of acetylpyrazole $\mathbf{1}$ with thiosemicarbazide $\mathbf{1 6}$ under MWI in ethanol containing drops of concentrated $\mathrm{HCl}$ led to the formation of product 19. Compound 19 was then react with 2-oxo$\mathrm{N}$-phenylpropanehydrazonoyl chloride (3a) in dioxane containing catalytic amount of TEA under MWI to give a product identical in all respects (IR, $\mathrm{mp}$ and mixed $\mathrm{mp}$.) with 18a (Scheme 3).

In a similar manner, when acetyl pyrazole $\mathbf{1}$ was allowed to react with thiosemicarbazide $\mathbf{2}$ and ethyl ( $N$-arylhydrazono)-chloroacetates $\mathbf{3 c}, \mathbf{f}$ in dioxane in the presence of triethylamine under MWI, it afforded in each case a single isolable product, namely, 2-(2-(1-(5-methyl1-(4-nitrophenyl)-3-(thiophen-2-yl)-1H-pyrazol-4-yl) ethylidene) hydrazinyl)-5-(2-arylhydrazono) thiazol4(5H)-one 21a, b (Scheme 4; Table 1). Structure 21 was confirmed by elemental analysis, spectral data (IR, ${ }^{1} \mathrm{H}$ NMR, and mass), and alternative synthesis route. Thus, thiosemicarbazone 19 was reacted with ethyl)-2-chloro2-(2-phenylhydrazono)acetate (3c) in dioxane in the presence of TEA under MWI afforded a product identical in all aspects (mp, mixed $\mathrm{mp}$, and spectra) with $\mathbf{2 1 a}$ (Scheme 4).

Finally, the reactivity of acetylpyrazole 1 towards hydrazonoyl halides, be bereft of a-keto group, was 


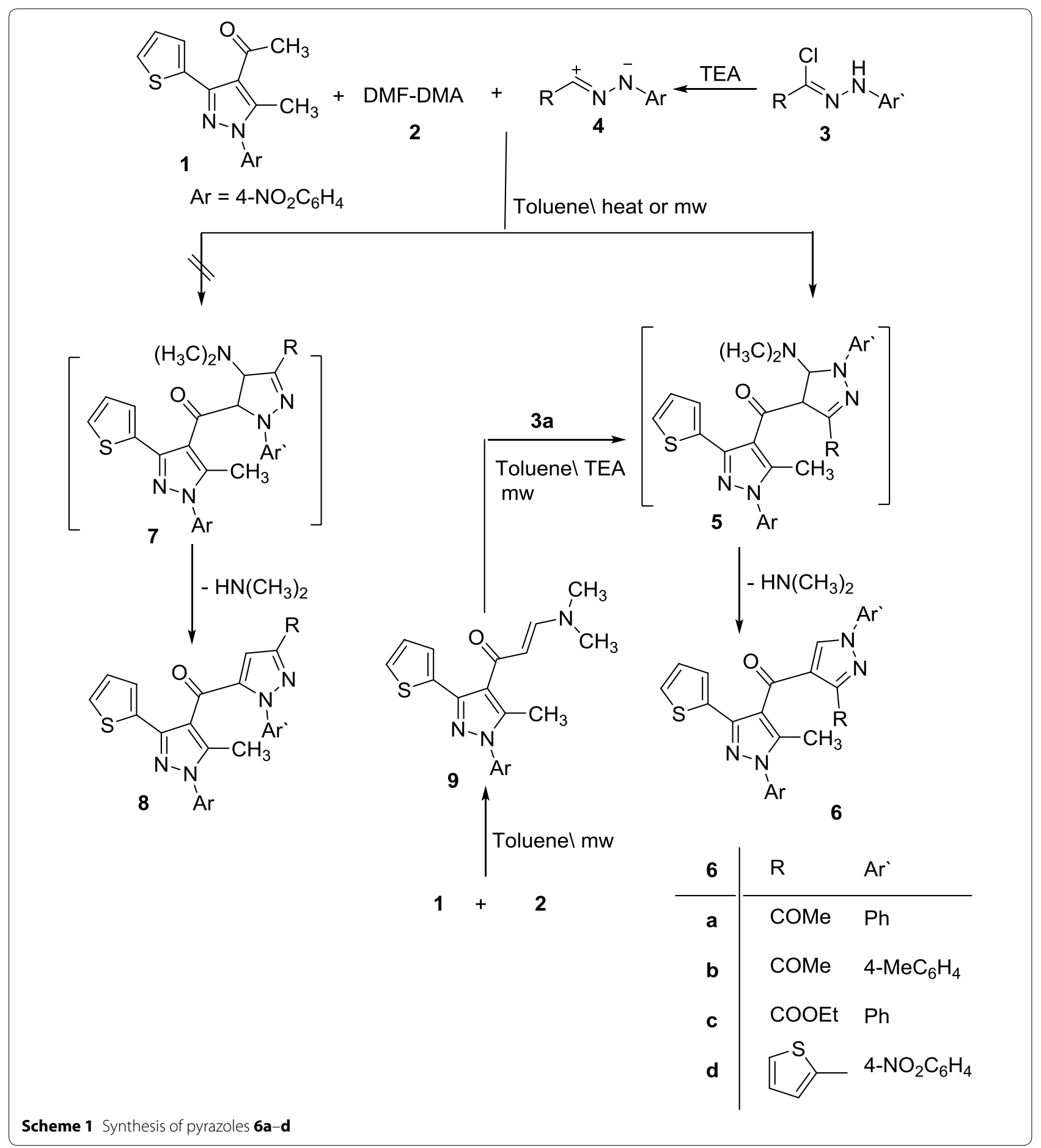

examined. In the present study, we have established that reaction of acetylpyrazole 1 with $N$-thiosemicarbazide $\mathbf{1 6}$ and aryl carbohydrazonoyl chlorides $\mathbf{3 d}$, g gave the respective 1,3,4-thiadiazoles $\mathbf{2 3 a}, \mathbf{b}$ as the end products (Scheme 5; Table 1). The structures of compounds 23a, b were confirmed on the bases of spectral data and elemental analyses (see Experimental part). The reaction proceeded via S-alkylation, with removal of hydrogen chloride, to give S-alkylated intermediates $\mathbf{2 2}$ followed by intramolecular Michael type addition under 
Table 1 Comparative data of conventional (A) and MW (B) methods for the synthesis of compounds $6 a-d, 13 a, b$, $18 a-c, 21 a, b$ and $23 a, b$

\begin{tabular}{|c|c|c|c|c|}
\hline \multirow[t]{2}{*}{ Compound no. } & \multicolumn{2}{|c|}{$\begin{array}{l}\text { Conventional } \\
\text { method }(A)\end{array}$} & \multicolumn{2}{|c|}{ Microwave method (B) } \\
\hline & Time (h) & Yield (\%) & Time (min) & Yield (\%) \\
\hline $6 a$ & 12 & 66 & 4 & 84 \\
\hline $6 b$ & 15 & 68 & 10 & 85 \\
\hline $6 c$ & 10 & 70 & 8 & 88 \\
\hline $6 d$ & 8 & 69 & 5 & 90 \\
\hline $13 a$ & 12 & 67 & 6 & 82 \\
\hline $13 b$ & 10 & 70 & 6 & 89 \\
\hline $18 \mathbf{a}$ & 8 & 66 & 7 & 90 \\
\hline $18 b$ & 6 & 68 & 10 & 88 \\
\hline $18 c$ & 4 & 67 & 7 & 90 \\
\hline $21 a$ & 6 & 69 & 8 & 86 \\
\hline $21 b$ & 5 & 64 & 6 & 92 \\
\hline $23 a$ & 8 & 72 & 10 & 81 \\
\hline $23 b$ & 8 & 67 & 9 & 83 \\
\hline
\end{tabular}

the employed reaction conditions, followed by elimination of ammonia, afforded the final product 23 [36, 47] (Scheme 5).

\section{Cytotoxic activity}

The in vitro growth inhibitory activity of the synthesized compounds $6 \mathbf{a}-\mathbf{d}, \mathbf{9}, 13 \mathbf{1}, \mathbf{b}, 18 \mathbf{a}-\mathbf{c}, 19,21 \mathbf{a}, \mathbf{b}$ and 23a, $\mathbf{b}$ was investigated against two carcinoma cell lines: human lung cancer (A-549) and human hepatocellular carcinoma(HepG-2) in comparison with the wellknown anticancer standard drug (cisplatin) under the same conditions using colorimetric MTT assay. Data generated were used to plot a dose response curve of which the concentration of test compounds required to kill $50 \%$ of cell population $\left(\mathrm{IC}_{50}\right)$ was determined. The results revealed that all the tested compounds showed inhibitory activity to the tumor cell lines in a concentration dependent manner. Interestingly, the results represented in Table 2 and Fig. 1 showed that compounds 13a, 13b and 21a were the most active compounds $\left(\mathrm{IC}_{50}\right.$ value of $4.47 \pm 0.3,3.46 \pm 0.6,3.10 \pm 0.8 \mu \mathrm{g} / \mathrm{mL}$, respectively) against the lung carcinoma cell line (A549), compared with cisplatin reference drug with $\mathrm{IC}_{50}$ value of $0.95 \pm 0.23 \mu \mathrm{g} / \mathrm{mL}$. Moreover, the order of activity against A549 cell line was $\mathbf{1 8 c}>\mathbf{1 8 b}>\mathbf{1 9}>\mathbf{9}>\mathbf{6 a}>\mathbf{6 c}$ $>23 b>6 d>18 a>21 b>6 b$.

On the other hand, compounds $6 \mathbf{a}, \mathbf{9}, \mathbf{1 3 b}, \mathbf{2 3 b}$ were the most active compounds $\left(\mathrm{IC}_{50}\right.$ value of $5.60 \pm 0.8$, $5.67 \pm 1.2,4.47 \pm 0.9$ and $5.67 \pm 1.2 \mu \mathrm{g} / \mathrm{mL}$, respectively) against liver carcinoma cell line (HepG2) cell line while the rest compounds have moderate activities.

\section{Experimental}

\section{Chemistry}

General

Melting points were measured on an Electrothermal IA 9000 series digital melting point apparatus (Bibby Sci. Lim. Stone, Staffordshire, UK). IR spectra were measured on PyeUnicam SP 3300 and Shimadzu FTIR 8101 PC infrared spectrophotometers (Shimadzu, Tokyo, Japan) in potassium bromide discs. NMR spectra were measured on a Varian Mercury VX-300 NMR spectrometer (Varian, Inc., Karlsruhe, Germany) operating at $300 \mathrm{MHz}\left({ }^{1} \mathrm{H}-\mathrm{NMR}\right)$ and run in deuterated dimethylsulfoxide $\left(\mathrm{DMSO}-d_{6}\right)$. Chemical shifts were related to that of the solvent. Mass spectra were recorded on a Shimadzu GCMS-QP1000 EX mass spectrometer (Tokyo, Japan) at $70 \mathrm{eV}$. Elemental analyses were measured by using a German made Elementarvario LIII CHNS analyzer. Antitumor activity of the products was measured at the Regional Center for Mycology and Biotechnology at Al-Azhar University, Cairo, Egypt. Hydrazonoyl halides 3a-g were prepared following literature method [41, 48].

\section{Synthetic procedures}

Synthesis of trisubstituted pyrazoles $6 a-d$ and isoxazoles $13 a, b$ Method $A$ To a stirred solution of acetyl pyrazole 1 (0.327 g, $1 \mathrm{mmol})$, dimethylformamide dimethylacetal 2 $(1 \mathrm{mmol})$ and the appropriate hydrazonoyl halides $\mathbf{3 a}-\mathbf{d}$ or hyroximoyl chlorides $\mathbf{1 0 a}, \mathbf{b}(1 \mathrm{mmol})$ in dry toluene (15 $\mathrm{mL})$, an equivalent amount of triethylamine $(0.5 \mathrm{~mL})$ was added. The reaction mixture was heated under reflux for 10-15 h (monitored through TLC). The precipitated triethylamine hydrochloride was filtered off, and the filtrate was evaporated under reduced pressure. The residue was triturated with $\mathrm{MeOH}$. The solid product, so formed in each case, was collected by filtration, washed with water, dried, and crystallized from the proper solvent to afford the corresponding pyrazole $\mathbf{6 a}-\mathbf{d}$ and isoxazole derivatives 13a, b, respectively.

Method B Repetition of the same reactions of method A with heating in microwave oven at $500 \mathrm{~W}$ and $150{ }^{\circ} \mathrm{C}$ for 4-10 min., gave products identical in all respects with those separated from method A. The products $\mathbf{6 a}-\mathbf{d}$ and 13a, b together with their physical constants are listed below.

1-(4-(5-Methyl-1-(4-nitrophenyl)-3-(thiophen-2-yl)-1Hpyrazole-4-carbonyl)-1-phenyl-1H-pyrazol-3-yl)ethanone (6a) Brown solid, mp 208-210 ${ }^{\circ} \mathrm{C}$; IR (KBr) $v_{\max } 1599$ $(\mathrm{C}=\mathrm{N}), 1670,1682(2 \mathrm{C}=\mathrm{O}), 2924,3105(\mathrm{C}-\mathrm{H}) \mathrm{cm}^{-1} ;{ }^{1} \mathrm{H}$ NMR (DMSO- $\left.d_{6}\right) \delta 2.34\left(\mathrm{~s}, 3 \mathrm{H}, \mathrm{CH}_{3}\right), 2.55\left(\mathrm{~s}, 3 \mathrm{H}, \mathrm{CH}_{3}\right)$, 6.98-8.39 (m, 12H, Ar-H), 8.92 (s, 1H, pyrazole-H5); MS m/z (\%) 497 (M $\left.\mathrm{M}^{+}, 9\right), 342$ (25), 252 (22), 174 (11), 145 (22), 


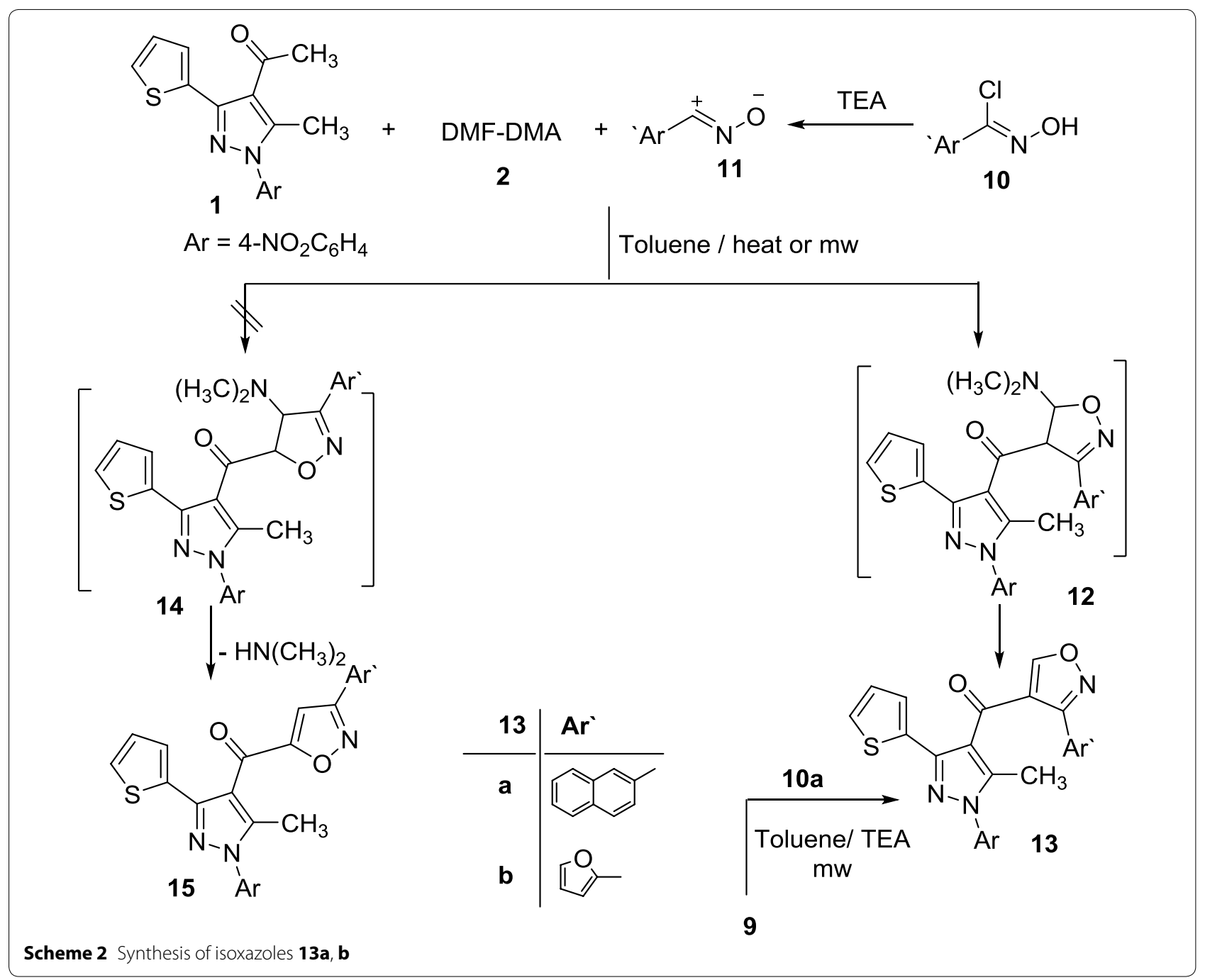

115 (26), 103 (40), 76 (100), 63 (13), 50 (19). Anal. Calcd. for $\mathrm{C}_{26} \mathrm{H}_{19} \mathrm{~N}_{5} \mathrm{O}_{4} \mathrm{~S}$ (497.53): C, 62.77; $\mathrm{H}, 3.85 ; \mathrm{N}, 14.08$. Found: C, 63.08; H, 3.55; N, 13.70\%.

1-(4-(5-Methyl-1-(4-nitrophenyl)-3-(thiophen-2-yl)-1H -pyrazole-4-carbonyl)-1-(p-tolyl)-1H-pyrazol-3-yl)ethanone (6b) Yellow solid, mp 222-224 ${ }^{\circ} \mathrm{C}$; IR (KBr) $v_{\max }$ $1597(\mathrm{C}=\mathrm{N}), 1676,1688(2 \mathrm{C}=\mathrm{O}), 2919,3118(\mathrm{C}-\mathrm{H})$ $\mathrm{cm}^{-1} ;{ }^{1} \mathrm{H}$ NMR (DMSO-d 6 ) $\delta 2.24\left(\mathrm{~s}, 3 \mathrm{H}, \mathrm{CH}_{3}\right), 2.34(\mathrm{~s}$, $\left.3 \mathrm{H}, \mathrm{CH}_{3}\right), 2.56\left(\mathrm{~s}, 3 \mathrm{H}, \mathrm{CH}_{3}\right), 7.12(\mathrm{t}, J=1.2 \mathrm{~Hz}, 1 \mathrm{H}$, thiophene-H), 7.31 (d, $J=1.2 \mathrm{~Hz}, 1 \mathrm{H}$, thiophene- $\mathrm{H}), 7.33$ (d, $J=1.2 \mathrm{~Hz}, 1 \mathrm{H}$, thiophene-H), $7.55(\mathrm{~d}, J=4.4 \mathrm{~Hz}, 2 \mathrm{H}$, Ar-H), $7.63(\mathrm{~d}, J=4.4 \mathrm{~Hz}, 2 \mathrm{H}, \mathrm{Ar}-\mathrm{H}), 7.88(\mathrm{~d}, J=8.8 \mathrm{~Hz}$, $2 \mathrm{H}, \mathrm{Ar}-\mathrm{H}), 8.39$ (d, $J=8.8 \mathrm{~Hz}, 2 \mathrm{H}, \mathrm{Ar}-\mathrm{H}), 10.58$ (s, $1 \mathrm{H}$, pyrazole-H5); ${ }^{13} \mathrm{C}$-NMR (DMSO- $\left.d_{6}\right): \delta 13.3,20.8,25.7$ $\left(\mathrm{CH}_{3}\right), 115.3,117.6,118.9,121.37,122.7,125.2,126.7$, $128.1,129.4,130.1,132.2,133.8,138.1,140.6,143.43$, 144.4, 146.8, 147.2 (Ar-C and $C=N), 188.2,194.9(C=O)$;
MS m/z (\%) $511\left(\mathrm{M}^{+}, 2\right), 406$ (10), 266 (6), 219 (11), 168 (7), 147 (7), 125 (11), 104 (25), 98 (17), 83 (93), 79 (44), 69 (35), 54 (53), 44 (100). Anal. Calcd. for $\mathrm{C}_{27} \mathrm{H}_{21} \mathrm{~N}_{5} \mathrm{O}_{4} \mathrm{~S}$ (511.55): C, 63.58; H, 4.14; N, 13.69. Found: C, 63.78; H, $4.05 ;$ N, $13.29 \%$.

Ethyl 4-(5-methyl-1-(4-nitrophenyl)-3-(thiophen-2-yl)1H-pyrazole-4-carbonyl)-1-phenyl-1H-pyrazole-3-carboxylate (6c) Yellow solid, mp 207-209 ${ }^{\circ} \mathrm{C}$; IR (KBr) $v_{\max } 15,984(\mathrm{C}=\mathrm{N}), 1660,1724(2 \mathrm{C}=\mathrm{O}), 2931,2974$ $(\mathrm{C}-\mathrm{H}) \mathrm{cm}^{-1} ;{ }^{1} \mathrm{H}$ NMR (DMSO-d 6 ) $\delta 1.18(\mathrm{t}, J=7.6 \mathrm{~Hz}$, $3 \mathrm{H}, \mathrm{CH}_{3} \mathrm{CH}_{2}$ ), $2.34\left(\mathrm{~s}, 3 \mathrm{H}, \mathrm{CH}_{3}\right), 4.27$ (q, $J=7.1 \mathrm{~Hz}, 2 \mathrm{H}$, $\left.\mathrm{CH}_{2} \mathrm{CH}_{3}\right), 6.96-8.43(\mathrm{~m}, 12 \mathrm{H}, \mathrm{Ar}-\mathrm{H}), 8.99$ (s, $1 \mathrm{H}$, pyrazole-H5); MS m/z (\%) $527\left(\mathrm{M}^{+}, 6\right), 484$ (22), 366 (26), 328 (33), 268 (50), 226 (35), 210 (37), 151 (49), 124 (78), 115 (61), 75 (100), 42 (45). Anal. Calcd. for $\mathrm{C}_{27} \mathrm{H}_{21} \mathrm{~N}_{5} \mathrm{O}_{5} \mathrm{~S}$ (527.55): C, 61.47; H, 4.01; N, 13.28. Found: C, 61.77; H, 3.75 ; N, $12.94 \%$. 


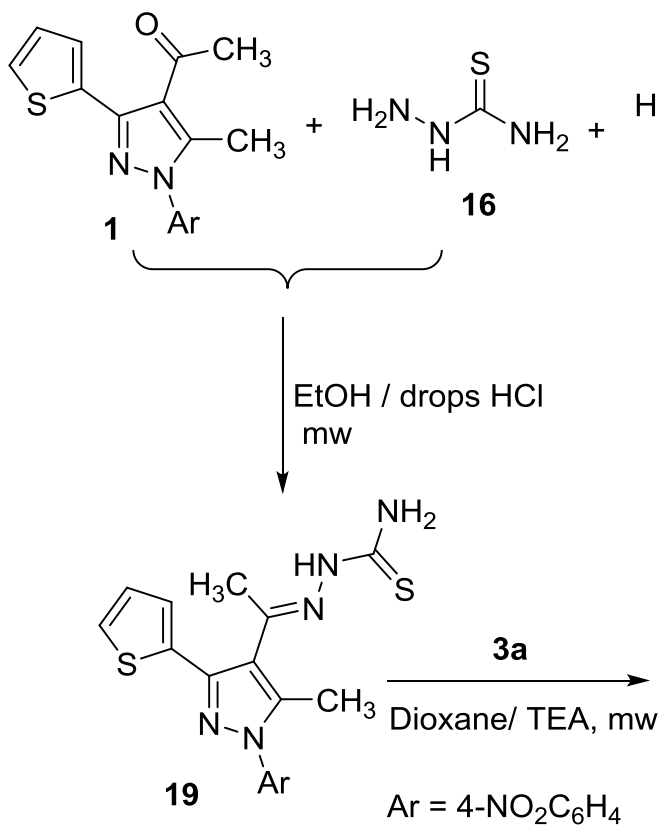

Scheme $\mathbf{3}$ Synthesis of thiazoles $\mathbf{1 8 a}-\mathbf{c}$

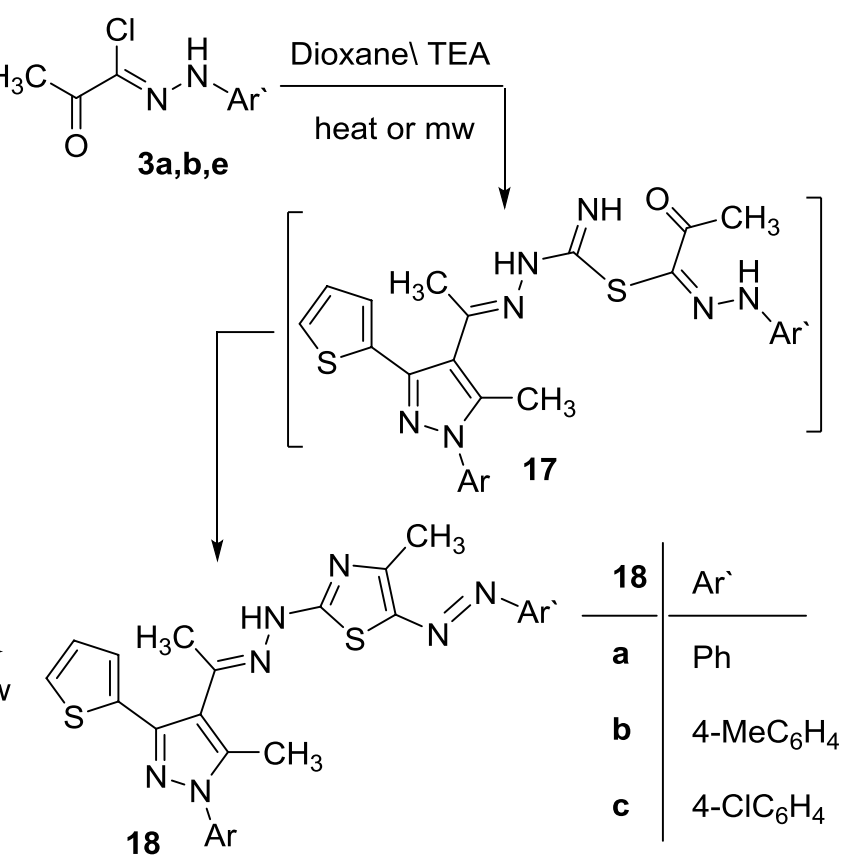<smiles>CCOC(=O)/C(Cl)=N/N[Al]</smiles>

16

Dioxane /TEA heat or $\mathrm{mw}$

$\mathrm{Ar}=4-\mathrm{NO}_{2} \mathrm{C}_{6} \mathrm{H}_{4}$

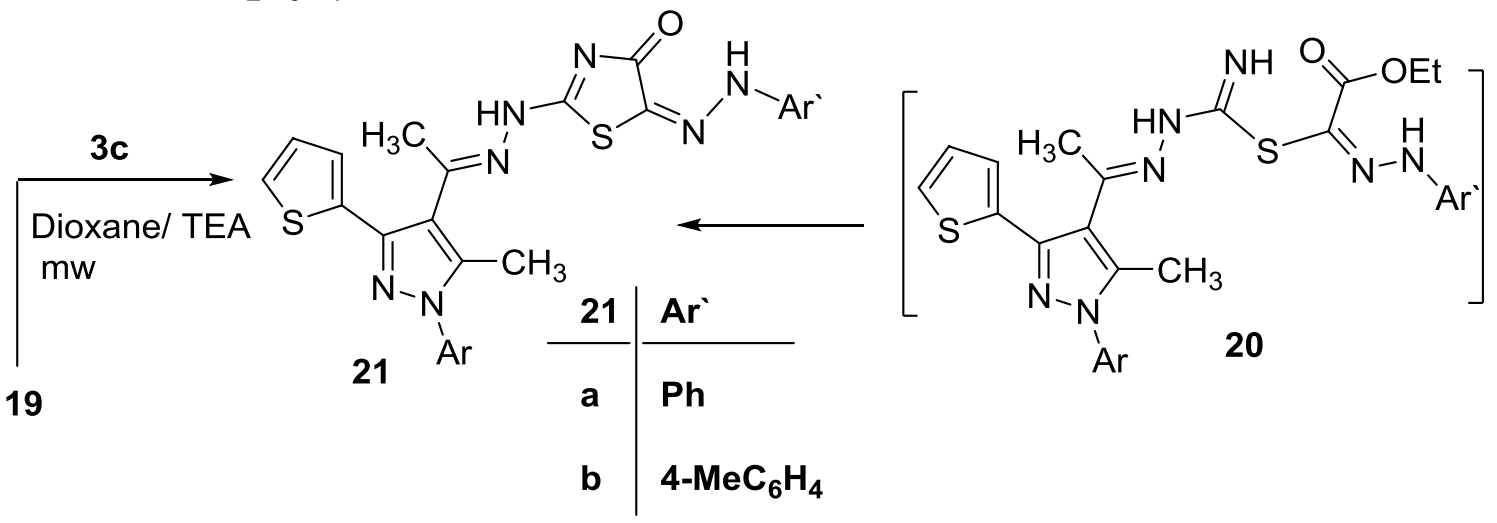

Scheme 4 Synthesis of thiazolones $\mathbf{2 1 a}, \mathbf{b}$

(5-Methyl-1-(4-nitrophenyl)-3-(thiophen-2-yl)-1H-pyrazol-4-yl)(1-(4-nitrophenyl)-3-(thiophen-2-yl)-1H-pyrazol-4-yl)methanone (6d) Orange solid, mp 219-220 ${ }^{\circ} \mathrm{C}$; IR (KBr) $v_{\max } 1595(\mathrm{C}=\mathrm{N}), 1638(\mathrm{C}=\mathrm{O}), 2924,3105(\mathrm{C}-\mathrm{H})$ $\mathrm{cm}^{-1} ;{ }^{1} \mathrm{H}$ NMR (DMSO- $\left.d_{6}\right) \delta 2.34\left(\mathrm{~s}, 3 \mathrm{H}, \mathrm{CH}_{3}\right), 6.98-8.52$ (m, 14H, Ar-H), 9.28 (s, 1H, pyrazole-H5); ${ }^{13} \mathrm{C}-\mathrm{NMR}$ (DMSO- $\left.d_{6}\right): \delta 26.9\left(\mathrm{CH}_{3}\right), 113.1,113.3,115.0,115.6,122.5$, 122.6, 123.1, 123.6, 126.5, 126.7, 128.4, 131.1, 131.7, 132.1, 


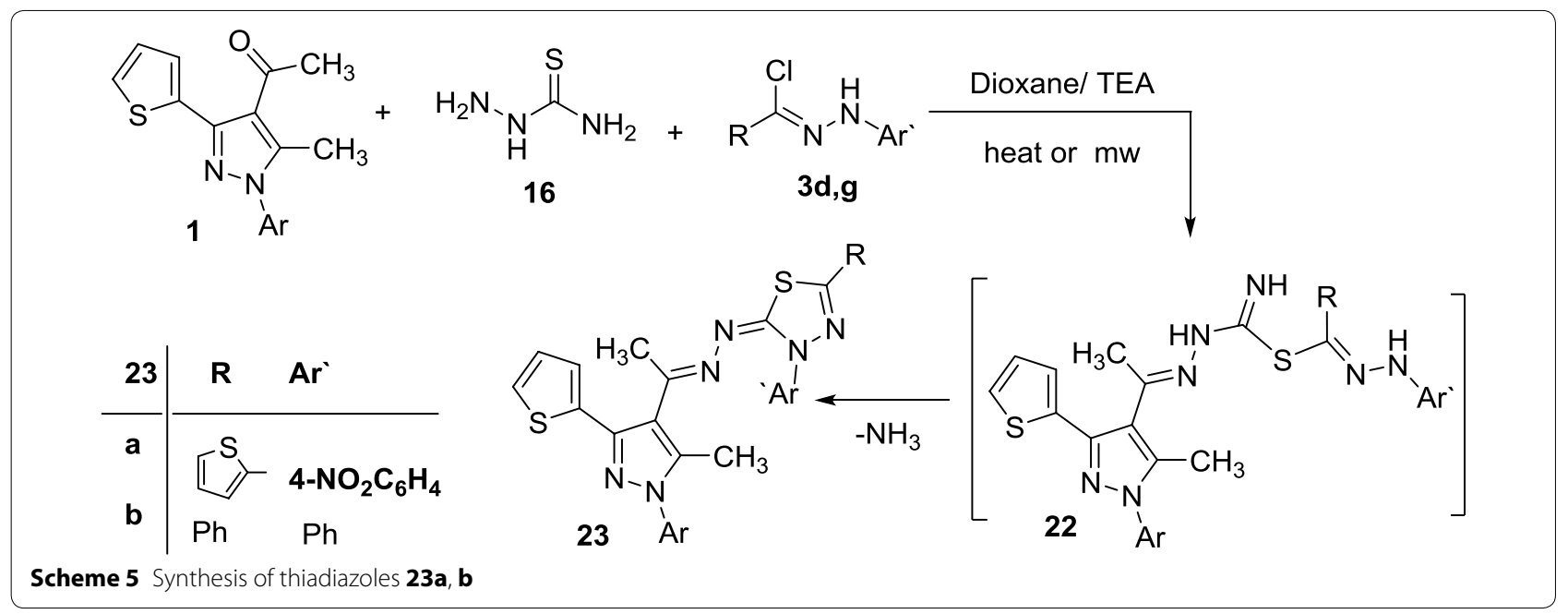

Table 2 The in vitro inhibitory activity of tested compounds against tumor cell lines expressed as IC50 values ( $\mu \mathrm{g} / \mathrm{mL}$ ) 土standard deviation from three replicates

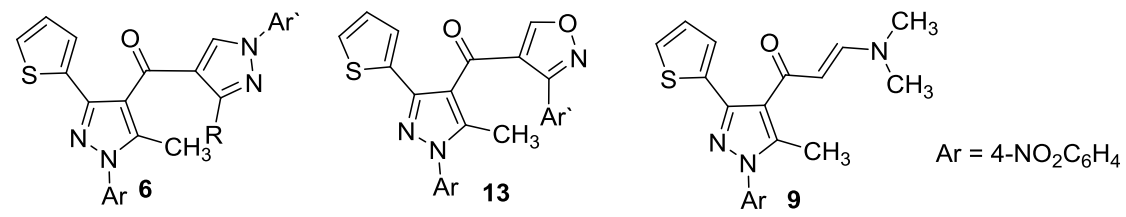

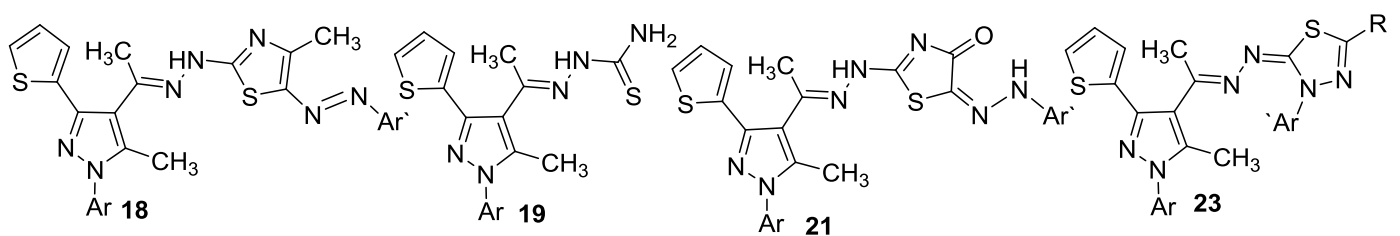

\begin{tabular}{|c|c|c|c|c|}
\hline \multirow[t]{2}{*}{ Tested compounds } & \multirow[t]{2}{*}{$\mathbf{R}$} & \multirow[t]{2}{*}{$A r^{\prime}$} & \multicolumn{2}{|c|}{ Tumor cell lines } \\
\hline & & & A-549 & HepG2 \\
\hline $6 a$ & $\mathrm{COCH}_{3}$ & $\mathrm{Ph}$ & $22.9 \pm 0.9$ & $5.60 \pm 0.8$ \\
\hline 6b & $\mathrm{COCH}_{3}$ & $4-\mathrm{MeC}_{6} \mathrm{H}_{4}$ & $38.5 \pm 1.2$ & $44.4 \pm 1.3$ \\
\hline $6 c$ & COOEt & $\mathrm{Ph}$ & $23.3 \pm 0.9$ & $22.4 \pm 0.9$ \\
\hline 6d & 2-Thienyl & $4-\mathrm{NO}_{2} \mathrm{C}_{6} \mathrm{H}_{4}$ & $30.6 \pm 1.1$ & $35.9 \pm 1.4$ \\
\hline 9 & - & - & $22.6 \pm 0.8$ & $5.67 \pm 1.2$ \\
\hline $13 a$ & - & 2-Naphthyl & $4.47 \pm 0.3$ & $8.03 \pm 1.1$ \\
\hline $13 \mathbf{b}$ & - & 2-Furyl & $3.46 \pm 0.6$ & $4.67 \pm 0.9$ \\
\hline $18 a$ & - & $\mathrm{Ph}$ & $32.7 \pm 1.2$ & $22.4 \pm 1.1$ \\
\hline $18 \mathrm{~b}$ & - & $4-\mathrm{MeC}_{6} \mathrm{H}_{4}$ & $19.1 \pm 1.1$ & $6.67 \pm 1.3$ \\
\hline $18 \mathrm{c}$ & - & $4-\mathrm{ClC}_{6} \mathrm{H}_{4}$ & $18.2 \pm 0.9$ & $21.8 \pm 0.9$ \\
\hline 19 & - & - & $21.3 \pm 0.8$ & $23.1 \pm 1.1$ \\
\hline $21 a$ & - & $\mathrm{Ph}$ & $3.10 \pm 0.8$ & $23.9 \pm 1.1$ \\
\hline $21 \mathrm{~b}$ & - & $4-\mathrm{MeC}_{6} \mathrm{H}_{4}$ & $33.6 \pm 0.9$ & $43.4 \pm 0.8$ \\
\hline $23 a$ & 2-Thienyl & 4- $\mathrm{NO}_{2} \mathrm{C}_{6} \mathrm{H}_{4}$ & $27.9 \pm 1.1$ & $34.4 \pm 0.9$ \\
\hline $23 b$ & $\mathrm{Ph}$ & $\mathrm{Ph}$ & $23.4 \pm 1.2$ & $5.67 \pm 1.7$ \\
\hline Cisplatin & - & - & $0.95 \pm 0.23$ & $1.4 \pm 0.37$ \\
\hline
\end{tabular}




\section{For the lung carcinoma cell line (A-549):}<smiles>Cc1c(-c2cccs2)nn(-c2ccc([N+](=O)[O-])cc2)c1C(=O)c1conc1-c1ccc2ccccc2c1</smiles>

$13 \mathrm{a}\left(\mathrm{IC}_{50}=4.47 \pm 0.3 \mu \mathrm{g} / \mathrm{mL}\right)$<smiles>Cc1c(C(=O)c2conc2-c2ccco2)c(-c2cccs2)nn1-c1ccc([N+](=O)[O-])cc1</smiles>

13b $\left(\mathrm{IC}_{50}=3.46 \pm 0.6 \mu \mathrm{g} / \mathrm{mL}\right)$

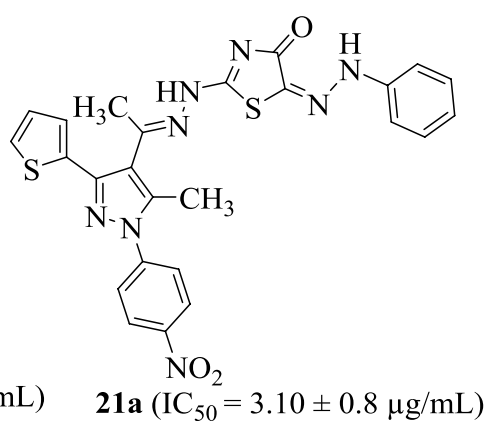

For the liver carcinoma cell line (HepG2):<smiles>CC(=O)c1nn(-c2ccccc2)cc1C(=O)c1c(-c2cccs2)nn(-c2ccc([N+](=O)[O-])cc2)c1C</smiles>

$\mathrm{NO}_{2}$

6a $\left(\mathrm{IC}_{50}=5.60 \pm 0.8 \mu \mathrm{g} / \mathrm{mL}\right)$<smiles>Cc1c(C(=O)/C=C/N(C)C)c(-c2cccs2)nn1-c1ccc([N+](=O)[O-])cc1</smiles><smiles>Cc1c(-c2cccs2)nn(-c2ccc([N+](=O)[O-])cc2)c1C(=O)c1conc1-c1ccco1</smiles>

13b $\left(\mathrm{IC}_{50}=4.47 \pm 0.9 \mu \mathrm{g} / \mathrm{mL}\right)$

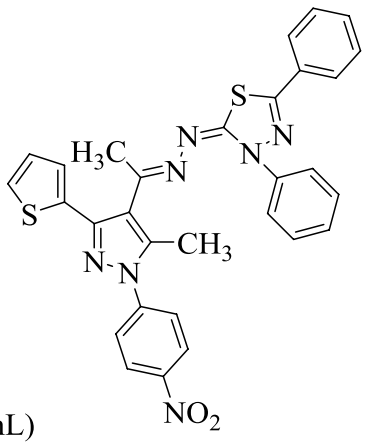

$9\left(\mathrm{IC}_{50}=5.67 \pm 1.2 \mu \mathrm{g} / \mathrm{mL}\right)$

23b $\left(\mathrm{IC}_{50}=5.67 \pm 1.2 \mu \mathrm{g} / \mathrm{mL}\right)$

Fig. 1 Cytotoxic activities of the most active compounds against HEPG2 and A-549 cell lines

132.3, 136.5, 137.1, 141.5, 141.6, 142.4, 142.6, 142.8 (Ar-C and $\mathrm{C}=\mathrm{N}), 197.2(\mathrm{C}=\mathrm{O}) ; \mathrm{MS} \mathrm{m} / \mathrm{z}(\%) 582\left(\mathrm{M}^{+}, 6\right), 532$ (12), 383 (16), 286 (11), 219 (21), 135 (49), 79 (16), 83 (27), 76 (67), 60 (28), 45 (100). Anal. Calcd. for $\mathrm{C}_{28} \mathrm{H}_{18} \mathrm{~N}_{6} \mathrm{O}_{5} \mathrm{~S}_{2}$ (582.61): C, 57.72; H, 3.11; N, 14.42. Found: C, 57.99; H, $2.80 ; \mathrm{N}, 14.12 \%$.

Synthesis of 3-(dimethylamino)-1-(5-methyl-1-(4-nitroph enyl)-3-(thiophen-2-yl)-1H-pyrazol-4-yl)prop-2-en-1-one (9). Amixture of acetyl pyrazole 1 (3.27 g, $10 \mathrm{mmol})$ and dimethylformamide-dimethylacetal (DMF-DMA) $(10 \mathrm{mmol})$ in dry toluene $(20 \mathrm{~mL})$ was refluxed in microwave oven at $500 \mathrm{~W}$ and $150{ }^{\circ} \mathrm{C}$ for $5 \mathrm{~min}$., then left to cool to room temperature. The precipitated product was filtered off, washed with light petroleum $\left(40-60{ }^{\circ} \mathrm{C}\right)$, and dried. Recrystallization from benzene afforded enaminone 1 as orange solid, $\mathrm{mp} 250-252{ }^{\circ} \mathrm{C}$; IR ( $\mathrm{KBr}$ ) $v_{\max } 1642(\mathrm{C}=\mathrm{O}), 2920,3080(\mathrm{C}-\mathrm{H}) \mathrm{cm}^{-1} ;{ }^{1} \mathrm{H}$ NMR (DMSO- $\left.d_{6}\right) \delta 2.34\left(\mathrm{~s}, 3 \mathrm{H}, \mathrm{CH}_{3}\right), 2.87\left(\mathrm{~s}, 3 \mathrm{H}, \mathrm{CH}_{3}\right), 3.06$ $\left(\mathrm{s}, 3 \mathrm{H}, \mathrm{CH}_{3}\right), 5.24(\mathrm{~d}, J=12.8 \mathrm{~Hz}, 1 \mathrm{H}, \mathrm{N}-\mathrm{CH}=), 7.05(\mathrm{t}$, $J=1.2 \mathrm{~Hz}, 1 \mathrm{H}$, thiophene- $\mathrm{H}), 7.14(\mathrm{~d}, J=1.2 \mathrm{~Hz}, 1 \mathrm{H}$, thiophene-H), $7.50(\mathrm{~d}, J=1.2 \mathrm{~Hz}, 1 \mathrm{H}$, thiophene- $\mathrm{H})$, $7.65(\mathrm{~d}, J=12.8 \mathrm{~Hz}, 1 \mathrm{H},=\mathrm{CH}-\mathrm{CO}), 7.90(\mathrm{~d}, J=8.8 \mathrm{~Hz}$, $2 \mathrm{H}, \mathrm{Ar}-\mathrm{H}), 8.37$ (d, $J=8.8 \mathrm{~Hz}, 2 \mathrm{H}, \mathrm{Ar}-\mathrm{H}) ;{ }^{13} \mathrm{C}-\mathrm{NMR}$ (DMSO- $\left.d_{6}\right): \delta 12.4,36.1,44.0\left(\mathrm{CH}_{3}\right), 120.4,124.3,124.4$,
$125.8,127.0,127.1,128.4,134.0,142.5,143.5,145.4$, 145.8, 146.3 (Ar-C and $\mathrm{C}=\mathrm{N}), 194.0(\mathrm{C}=\mathrm{O}) ; \mathrm{MS} \mathrm{m} / \mathrm{z}$ (\%) $382\left(\mathrm{M}^{+}, 3\right), 300$ (11), 286 (11), 189 (9), 132 (7), 104 (100), 77 (58), 64 (16), 51 (13), 43 (12). Anal. Calcd. for $\mathrm{C}_{19} \mathrm{H}_{18} \mathrm{~N}_{4} \mathrm{O}_{3} \mathrm{~S}$ (382.44): C, 59.67; H, 4.74; $\mathrm{N}, 14.65$. Found: C, 59.58; H, 4.44; N, 14.39\%.

(5-Methyl-1-(4-nitrophenyl)-3-(thiophen-2-yl)-1H-pyrazol-4-yl)(3-(naphthalen-2-yl)isoxazol-4-yl)methanone (13a) Yellow solid, mp 203-205 ${ }^{\circ} \mathrm{C}$; IR (KBr) $v_{\max } 1597$ $(\mathrm{C}=\mathrm{N}), 1660(\mathrm{C}=\mathrm{O}), 2976,3117(\mathrm{C}-\mathrm{H}) \mathrm{cm}^{-1}$; ${ }^{1} \mathrm{H}$ NMR $\left(\mathrm{DMSO}-d_{6}\right) \delta 2.31\left(\mathrm{~s}, 3 \mathrm{H}, \mathrm{CH}_{3}\right), 7.13-8.45(\mathrm{~m}, 14 \mathrm{H}$, $\mathrm{Ar}-\mathrm{H}), 9.67$ (s, $1 \mathrm{H}$, isoxazole-H5); ${ }^{13} \mathrm{C}-\mathrm{NMR}$ (DMSO- $d_{6}$ ): $\delta 26.9\left(\mathrm{CH}_{3}\right), 110.0,113.3,115.0,115.1,115.5,122.5,123.3$, $124.5,125.0,126.5,126.7,128.4,130.8,133.6,135.4,136.9$, 137.0, 141.5, 141.6, 142.6, 148.8, 152.4, 160.0 (Ar-C and $\mathrm{C}=\mathrm{N}), 188.3(\mathrm{C}=\mathrm{O}) ; \mathrm{MS} \mathrm{m} / \mathrm{z}(\%) 506\left(\mathrm{M}^{+}, 2\right), 435(9), 412$ (14), 379 (45), 214 (12), 142 (10), 105 (26), 93 (21), 77 (51), 65 (62), 60 (52), 43 (100). Anal. Calcd. for $\mathrm{C}_{28} \mathrm{H}_{18} \mathrm{~N}_{4} \mathrm{O}_{4} \mathrm{~S}$ (506.53): C, 66.39; H, 3.58; N, 11.06. Found: C, 66.04; H, $3.21 ; \mathrm{N}, 10.86 \%$.

(3-(Furan-3-yl)isoxazol-4-yl)(5-methyl-1-(4-nitroph enyl)-3-(thiophen-2-yl)-1H-pyrazol-4-yl)methanone (13b) Orange solid, mp 209-211 ${ }^{\circ} \mathrm{C}$; IR (KBr) $v_{\max } 1598$ 
$(\mathrm{C}=\mathrm{N}), 1664(\mathrm{C}=\mathrm{O}), 2925,3107(\mathrm{C}-\mathrm{H}) \mathrm{cm}^{-1} ;{ }^{1} \mathrm{H}$ NMR $\left(\mathrm{DMSO}-d_{6}\right) \delta 2.34\left(\mathrm{~s}, 3 \mathrm{H}, \mathrm{CH}_{3}\right), 7.13-8.61(\mathrm{~m}, 10 \mathrm{H}$, $\mathrm{Ar}-\mathrm{H}), 9.23$ (s, 1H, pyrazole-H5); MS m/z (\%) $446\left(\mathrm{M}^{+}\right.$, 2), 392 (100), 349 (43), 317 (23), 285 (11), 234 (16), 191 (16), 172 (20), 130 (26), 102 (26), 77 (69). Anal. Calcd. for $\mathrm{C}_{22} \mathrm{H}_{14} \mathrm{~N}_{4} \mathrm{O}_{5} \mathrm{~S}$ (446.44): C, 59.19; H, 3.16; N, 12.55. Found: C, 59.50; H, 2.80; N, 12.17\%.

Alternate synthesis of $6 \boldsymbol{a}$ and $13 \boldsymbol{a}$ Equimolar amounts of enaminone 9 (0.382 g, $1 \mathrm{mmol})$ and hydrazonoyl halide 3a or hyroximoyl chloride $\mathbf{1 0 a}(1 \mathrm{mmol})$ in dry toluene $(15 \mathrm{~mL})$ containing an equivalent amount of triethylamine $(0.5 \mathrm{~mL})$ was refluxed in microwave oven at $500 \mathrm{~W}$ and $150{ }^{\circ} \mathrm{C}$ for 6 min., gave products identical in all respects (mp, mixed mp and IR spectra) with compounds $\mathbf{6 a}$ and 13a, respectively.

Synthesis of thiazoles 18a-c and 21a, b and thiadiazoles 23a, $b$ : Method $A$ To a stirred solution of acetyl pyrazole 1 (0.327 g, $1 \mathrm{mmol})$, thiosemicarbazide 16 (0.091 g, $1 \mathrm{mmol}$ ) and the appropriate hydrazonoyl halides $\mathbf{3 a}, \mathbf{b}, \mathbf{e}$ or 3c, f or 3d, $\mathbf{g}$ (1 mmol) in dioxane $(15 \mathrm{~mL})$, an equivalent amount of triethylamine $(0.05 \mathrm{~mL})$ was added. The reaction mixture was heated under reflux for 4-8 h (monitored through TLC). Excess of solvent was removed under reduced pressure and the reaction mixture was triturated with $\mathrm{MeOH}$. The product separated was filtered, washed with $\mathrm{MeOH}$, dried and recrystallized from the proper solvent to give thiazoles $18 \mathbf{a}-\mathbf{c}$ and $21 \mathbf{a}, \mathbf{b}$ and thiadiazoles $23 a, b$, respectively.

Method B Repetition of the same reactions of method A with heating in microwave oven at $500 \mathrm{~W}$ and $150{ }^{\circ} \mathrm{C}$ for 4-10 min., gave products identical in all respects with those separated from method A. The products $18 \mathbf{a}-\mathbf{c}$, 21a, b and 23a, b together with their physical constants are listed below.

4-Methyl-2-(2-(1-(5-methyl-1-(4-nitrophenyl)3-(thiophen-2-yl)-1H-pyrazol-4-yl)ethylidene) hydrazinyl)-5-(phenyldiazenyl)thiazole (18a) Orange solid, mp 219-220 ${ }^{\circ} \mathrm{C}$; IR (KBr) $v_{\max } 1600(\mathrm{C}=\mathrm{N}), 2974$ $(\mathrm{C}-\mathrm{H}), 3396(\mathrm{NH}) \mathrm{cm}^{-1}$; ${ }^{1} \mathrm{H}$ NMR (DMSO-d $) \delta 2.32(\mathrm{~s}$, $\left.3 \mathrm{H}, \mathrm{CH}_{3}\right), 2.36\left(\mathrm{~s}, 3 \mathrm{H}, \mathrm{CH}_{3}\right), 2.48\left(\mathrm{~s}, 3 \mathrm{H}, \mathrm{CH}_{3}\right), 6.99-7.93$ (m, 12H, Ar-H), 10.65 (s, 1H, NH); ${ }^{13} \mathrm{C}-\mathrm{NMR}$ (DMSO$\left.d_{6}\right): \delta 9.2,12.5,24.6\left(\mathrm{CH}_{3}\right), 114.5,121.4,123.1,125.2$, 126.3, 127.0, 127.9, 128.1, 128.5, 128.9, 135.3, 140.4, 140.9, 143.1, 144.1, 145.3, 145.79, 153.3, 163.4 (Ar-C and $C=N)$; MS m/z (\%) $542\left(\mathrm{M}^{+}, 6\right), 432$ (16), 253 (13), 138 (11), 106 (69), 90 (12), 78 (100), 64 (11), 51 (34). Anal. Calcd. for $\mathrm{C}_{26} \mathrm{H}_{22} \mathrm{~N}_{8} \mathrm{O}_{2} \mathrm{~S}_{2}$ (542.64): C, 57.55; H, 4.09; N, 20.65. Found: C, 57.87 ; H, 3.70; N, 20.35\%.
4-Methyl-2-(2-(1-(5-methyl-1-(4-nitrophenyl)-3-(thiophe n-2-yl)-1H-pyrazol-4-yl)ethylidene) hydrazinyl)-5-(p-tolyldiazenyl)thiazole (18b). Orange solid, mp 226-228 ${ }^{\circ} \mathrm{C}$; IR (KBr) $v_{\max } 1600(\mathrm{C}=\mathrm{N}), 2924(\mathrm{C}-\mathrm{H}), 3438(\mathrm{NH}) \mathrm{cm}^{-1}$; ${ }^{1} \mathrm{H}$ NMR (DMSO- $\left.d_{6}\right) \delta 2.17\left(\mathrm{~s}, 3 \mathrm{H}, \mathrm{CH}_{3}\right), 2.32(\mathrm{~s}, 3 \mathrm{H}$, $\left.\mathrm{CH}_{3}\right), 2.36\left(\mathrm{~s}, 3 \mathrm{H}, \mathrm{CH}_{3}\right), 2.47\left(\mathrm{~s}, 3 \mathrm{H}, \mathrm{CH}_{3}\right), 6.99-7.89(\mathrm{~m}$, $11 \mathrm{H}, \mathrm{Ar}-\mathrm{H}), 10.65$ (s, 1H, NH); ${ }^{13} \mathrm{C}-\mathrm{NMR}$ (DMSO- $d_{6}$ ): $\delta 12.0,14.3,15.7,26.8\left(\mathrm{CH}_{3}\right), 105.3,111.5,114.9,116.3$, $117.9,119.8,120.8,122.2,126.4,126.6,127.9,128.1,131.9$, 132.6, 137.6, 141.7, 142.1, 142.3, $170.2(\mathrm{Ar}-\mathrm{C}$ and $\mathrm{C}=\mathrm{N})$; MS m/z (\%) $556\left(\mathrm{M}^{+}, 18\right), 431$ (18), 314 (25), 251 (43), 193 (32), 166 (29), 152 (43), 136 (20), 119 (45), 104 (67), 90 (68), 75 (100), 62 (55), 52 (28), 41 (41). Anal. Calcd. for $\mathrm{C}_{27} \mathrm{H}_{24} \mathrm{~N}_{8} \mathrm{O}_{2} \mathrm{~S}_{2}$ (556.66): C, 58.26; H, 4.35; N, 20.13. Found: C, 58.58; H, 4.05; N, 19.80\%.

5-((4-Chlorophenyl)diazenyl)-4-methyl-2-(2-(1-(5-meth $y l$-1-(4-nitrophenyl)-3-(thiophen-2-yl)-1H-pyrazol-4-yl) ethylidene)hydrazinyl)thiazole (18c) Orange solid, $\mathrm{mp}$ 232-235 ${ }^{\circ} \mathrm{C}$; IR (KBr) v $v_{\max } 1598(\mathrm{C}=\mathrm{N}), 2922(\mathrm{C}-\mathrm{H}), 3436$ $(\mathrm{NH}) \mathrm{cm}^{-1} ;{ }^{1} \mathrm{H}$ NMR (DMSO- $\left.d_{6}\right) \delta 2.32\left(\mathrm{~s}, 3 \mathrm{H}, \mathrm{CH}_{3}\right), 2.36$ (s, 3H, $\left.\mathrm{CH}_{3}\right), 2.47$ (s, 3H, $\left.\mathrm{CH}_{3}\right), 6.99-7.93(\mathrm{~m}, 11 \mathrm{H}, \mathrm{Ar}-\mathrm{H})$, 10.65 (s, 1H, NH); ${ }^{13} \mathrm{C}-\mathrm{NMR}$ (DMSO- $d_{6}$ ): $\delta 12.2,19.1,24.7$ $\left(\mathrm{CH}_{3}\right), 120.3,125.1,125.3,125.4,127.0,127.1,127.2,128.2$, $128.4,134.3,140.3,140.4,143.9,144.1,144.2,145.5,146.3$, $146.4,170.4(\mathrm{Ar}-\mathrm{C}$ and $\mathrm{C}=\mathrm{N}) ; \mathrm{MS} \mathrm{m} / \mathrm{z}(\%) 579\left(\mathrm{M}^{+}+2,2\right)$, $577\left(\mathrm{M}^{+}, 5\right), 548$ (7), $378(14), 333$ (11), 271 (100), $211(20)$, 181 (20), 153 (18), 118 (16), 104 (66), 94 (36), 77 (52), 69 (36), 57 (37). Anal. Calcd. for $\mathrm{C}_{26} \mathrm{H}_{21} \mathrm{~N}_{8} \mathrm{ClO}_{2} \mathrm{~S}_{2}$ (577.08): C, 54.11; H, 3.67; N, 19.42. Found: C, 54.44; H, 3.35; N, $19.12 \%$.

Synthesis of 2-(1-(5-methyl-1-(4-nitrophenyl)-3-(thioph en-2-yl)-1H-pyrazol-4-yl)ethylidene) hydrazinecarbothioamide (19) Amixture of acetyl pyrazole 1 (3.27 g, $10 \mathrm{mmol})$ and thiosemicarbazide $16(0.91 \mathrm{~g}, 10 \mathrm{mmol})$ in ethanol $(20 \mathrm{~mL})$ containing catalytic amounts of concentrated $\mathrm{HCl}$ was refluxed in microwave oven at $500 \mathrm{~W}$ and $150{ }^{\circ} \mathrm{C}$ for $6 \mathrm{~min}$., then left to cool to room temperature. The precipitated product was filtered off, washed with ethanol, and dried. Recrystallization from acetic acid afforded thiosemicarbazone 19 as yellow solid, (78\% yield), mp 212-215 ${ }^{\circ} \mathrm{C}$; IR ( $\left.\mathrm{KBr}\right) v_{\max } 1596(\mathrm{C}=\mathrm{N}), 2926$ $(\mathrm{C}-\mathrm{H}), 3157,3241,3388\left(\mathrm{NH}\right.$ and $\left.\mathrm{NH}_{2}\right) \mathrm{cm}^{-1} ;{ }^{1} \mathrm{H}$ NMR $\left(\mathrm{DMSO}-d_{6}\right) \delta 2.17\left(\mathrm{~s}, 3 \mathrm{H}, \mathrm{CH}_{3}\right), 2.34\left(\mathrm{~s}, 3 \mathrm{H}, \mathrm{CH}_{3}\right), 7.10$ $(\mathrm{t}, J=1.2 \mathrm{~Hz}, 1 \mathrm{H}$, thiophene-H), $7.23(\mathrm{~d}, J=1.2 \mathrm{~Hz}, 1 \mathrm{H}$, thiophene-H), $7.56(\mathrm{~d}, J=1.2 \mathrm{~Hz}, 1 \mathrm{H}$, thiophene-H), $7.86(\mathrm{~d}, J=8.8 \mathrm{~Hz}, 2 \mathrm{H}, \mathrm{Ar}-\mathrm{H}), 8.20\left(\mathrm{~s}, 2 \mathrm{H}, \mathrm{NH}_{2}\right), 8.38(\mathrm{~d}$, $J=8.8 \mathrm{~Hz}, 2 \mathrm{H}, \mathrm{Ar}-\mathrm{H}), 10.28$ (s, 1H, NH); MS m/z (\%) 400 $\left(\mathrm{M}^{+}, 8\right), 322$ (21), 284 (30), 211 (18), 176 (24), 150 (26), 130 (25), 112 (29), 105 (71), 97 (40), 83 (45), 69 (63), 57 (62), 43 (100). Anal. Calcd. for $\mathrm{C}_{17} \mathrm{H}_{16} \mathrm{~N}_{6} \mathrm{O}_{2} \mathrm{~S}_{2}$ (400.48): C, 50.98; H, 4.03; N, 20.98. Found: C, 51.30; H, 3.73; N, 20.65\%. 
2-(2-(1-(5-Methyl-1-(4-nitrophenyl)-3-(thiophen-2-yl)-1H -pyrazol-4-yl)ethylidene) hydrazinyl)-5-(2-phenylhydrazono)thiazol-4(5H)-one (21a) Orange solid, $\mathrm{mp} 203-$ $205{ }^{\circ} \mathrm{C}$; IR $(\mathrm{KBr}) v_{\max } 1600(\mathrm{C}=\mathrm{N}), 1680(\mathrm{C}=\mathrm{O}), 2932$ $(\mathrm{C}-\mathrm{H}), 3211,3420(2 \mathrm{NH}) \mathrm{cm}^{-1} ;{ }^{1} \mathrm{H}$ NMR (DMSO- $\left.d_{6}\right) \delta$ $2.24\left(\mathrm{~s}, 3 \mathrm{H}, \mathrm{CH}_{3}\right), 2.42\left(\mathrm{~s}, 3 \mathrm{H}, \mathrm{CH}_{3}\right), 7.12-7.92(\mathrm{~m}, 12 \mathrm{H}$, Ar-H), 9.82 (s, 1H, NH), 10.27 (s, 1H, NH); ${ }^{13} \mathrm{C}-\mathrm{NMR}$ (DMSO- $\left.d_{6}\right): \delta 12.1,23.2\left(\mathrm{CH}_{3}\right), 112.6,120.9,125.3,125.6$, 125.9, 127.0, 127.3, 127.8, 128.2, 128.4, 134.3, 140.2, 140.4, 143.1, 144.7, 145.2, 155.5, $160.1(\mathrm{Ar}-\mathrm{C}$ and $\mathrm{C}=\mathrm{N}), 175.4$ $(\mathrm{C}=\mathrm{O}) ; \mathrm{MS} \mathrm{m} / \mathrm{z}(\%) 544\left(\mathrm{M}^{+}, 3\right), 367$ (18), 267 (15), 194 (17), 177 (18), 129 (25), 115 (29), 102 (38), 91 (39), 79 (35), 72 (93), 60 (100), 43 (71). Anal. Calcd. for $\mathrm{C}_{25} \mathrm{H}_{20} \mathrm{~N}_{8} \mathrm{O}_{3} \mathrm{~S}_{2}$ (544.61): C, 55.13; H, 3.70; N, 20.58. Found: C, 55.44; H, $3.40 ; \mathrm{N}, 20.25 \%$.

2-(2-(1-(5-Methyl-1-(4-nitrophenyl)-3-(thiophen-2-yl)-1H -pyrazol-4-yl)ethylidene) hydrazinyl)-5-(2-(p-tolyl)hydrazono)thiazol-4(5H)-one (21b) Orange solid, mp 201$203{ }^{\circ} \mathrm{C}$; IR $(\mathrm{KBr}) v_{\max } 1596(\mathrm{C}=\mathrm{N}), 1675(\mathrm{C}=\mathrm{O}), 2920$, $2978(\mathrm{C}-\mathrm{H}), 3272,3419(2 \mathrm{NH}) \mathrm{cm}^{-1}$; ${ }^{1} \mathrm{H}$ NMR (DMSO$\left.d_{6}\right) \delta 2.28\left(\mathrm{~s}, 3 \mathrm{H}, \mathrm{CH}_{3}\right), 2.35\left(\mathrm{~s}, 3 \mathrm{H}, \mathrm{CH}_{3}\right), 2.48\left(\mathrm{~s}, 3 \mathrm{H}, \mathrm{CH}_{3}\right)$, 6.94-8.43 (m, 11H, Ar-H), $10.51(\mathrm{~s}, 1 \mathrm{H}, \mathrm{NH}), 10.54(\mathrm{~s}$, $1 \mathrm{H}, \mathrm{NH}) ;{ }^{13} \mathrm{C}-\mathrm{NMR}$ (DMSO- $\left.d_{6}\right): \delta 13.5,14.5,21.1\left(\mathrm{CH}_{3}\right)$, 112.0, 114.9, 116.3, 117.5, 119.5, 122.2, 125.3, 126.6, 128.0, 129.8, 136.5, 137.4, 138.4, 142.1, 148.2, 151.8, 154.5, 160.1 $(\mathrm{Ar}-\mathrm{C}$ and $\mathrm{C}=\mathrm{N}), 173.5(\mathrm{C}=\mathrm{O})$; $\mathrm{MS} \mathrm{m} / \mathrm{z}(\%) 558\left(\mathrm{M}^{+}, 2\right)$, 536 (11), 457 (61), 423 (12), 396 (27), 284 (44), 212 (45), 187 (51), 158 (22), 145 (36), 115 (57), 95 (41), 65 (100), 51 (28). Anal. Calcd. for $\mathrm{C}_{26} \mathrm{H}_{22} \mathrm{~N}_{8} \mathrm{O}_{3} \mathrm{~S}_{2}$ (558.63): C, 55.90; H, 3.97; N, 20.06. Found: C, 56.20; H, 3.65; N, 19.70\%.

2-((1-(5-Methyl-1-(4-nitrophenyl)-3-(thiophen-2-yl)-1Hpyrazol-4-yl)ethylidene)hydrazono)-3,5-diphenyl-2,3-dihydro-1,3,4-thiadiazole (23a) Orange solid, mp 195$197^{\circ} \mathrm{C}$; IR (KBr) $v_{\max } 1591(\mathrm{C}=\mathrm{N}), 2924,3105(\mathrm{C}-\mathrm{H}) \mathrm{cm}^{-1}$; ${ }^{1} \mathrm{H} \mathrm{NMR}\left(\mathrm{DMSO}-d_{6}\right) \delta 2.18\left(\mathrm{~s}, 3 \mathrm{H}, \mathrm{CH}_{3}\right), 2.43\left(\mathrm{~s}, 3 \mathrm{H}, \mathrm{CH}_{3}\right)$, 7.09-8.42 (m, $17 \mathrm{H}, \mathrm{Ar}-\mathrm{H}) ;{ }^{13} \mathrm{C}-\mathrm{NMR}$ (DMSO- $\left.d_{6}\right): \delta 12.1$, $24.7\left(\mathrm{CH}_{3}\right), 113.6,120.3,122.1,125.3,125.9,126.0,127.5$, 127.8, 128.2, 128.4, 130.2, 133.5, 134.3, 135.3, 137.3, 140.4, 143.1, 144.4, 145.5, 146.3, 146.4, 159.4 ( $\mathrm{Ar}-\mathrm{C}$ and $\mathrm{C}=\mathrm{N})$; MS m/z (\%) 577 (M+ $\left.\mathrm{M}^{+}, 6\right), 492$ (36), 441 (20), 356 (30), 327 (59), 269 (42), 177 (57), 121 (51), 103 (100), 77 (77), 55 (72), 42 (30). Anal. Calcd. for $\mathrm{C}_{30} \mathrm{H}_{23} \mathrm{~N}_{7} \mathrm{O}_{2} \mathrm{~S}_{2}$ (577.68): C, 62.37; $\mathrm{H}, 4.01 ; \mathrm{N}, 16.97$. Found: $\mathrm{C}, 62.68 ; \mathrm{H}, 3.70 ; \mathrm{N}, 16.62 \%$.

2-((1-(5-Methyl-1-(4-nitrophenyl)-3-(thiophen-2-yl)1H-pyrazol-4-yl)ethylidene) hydrazono)-3-(4-nitroph enyl)-5-(thiophen-3-yl)-2,3-dihydro-1,3,4-thiadiazole (23b) Orange solid, mp 209-210 ${ }^{\circ} \mathrm{C}$; IR (KBr) $v_{\max } 1693$ $(\mathrm{C}=\mathrm{N}), 2954(\mathrm{C}-\mathrm{H}) \mathrm{cm}^{-1} ;{ }^{1} \mathrm{H}$ NMR (DMSO- $\left.d_{6}\right) \delta 2.18(\mathrm{~s}$, $\left.3 \mathrm{H}, \mathrm{CH}_{3}\right), 2.27\left(\mathrm{~s}, 3 \mathrm{H}, \mathrm{CH}_{3}\right), 7.10-8.42(\mathrm{~m}, 14 \mathrm{H}, \mathrm{Ar}-\mathrm{H})$; $\mathrm{MS}$ $\mathrm{m} / \mathrm{z}(\%) 628\left(\mathrm{M}^{+}, 7\right), 561$ (11), 510 (31), 441 (20), 360 (26), 313 (24), 284 (78), 270 (52), 190 (26), 152 (100), 105 (63), 89 (30), 63 (39). Anal. Calcd. for $\mathrm{C}_{28} \mathrm{H}_{20} \mathrm{~N}_{8} \mathrm{O}_{4} \mathrm{~S}_{3}$ (628.70): C, 53.49; H, 3.21; N, 17.82. Found: C, 53.81; H, 2.90; N, 17.51\%.

Alternate synthesis of thiazole $18 a$ and $21 a$ Equimolar amounts of thiosemicarbazone $19(0.400 \mathrm{~g}, 1 \mathrm{mmol})$ and hydrazonoyl chloride $3 \mathbf{a}$ or $3 \mathbf{c}(1 \mathrm{mmol})$ in dioxane $(15 \mathrm{~mL})$ containing an equivalent amount of triethylamine $(0.05 \mathrm{~mL})$ was refluxed in microwave oven at $500 \mathrm{~W}$ and $150{ }^{\circ} \mathrm{C}$ for $3 \mathrm{~min}$., gave product identical in all respects (mp, mixed mp and IR spectra) with compounds 18a and 21a, respectively.

\section{Biological activity Anticancer activity}

The cytotoxic evaluation of the synthesized compounds was carried out at the Regional Center for Mycology and Biotechnology at Al-Azhar University, Cairo, Egypt according to the reported method [49].

\section{Conclusion}

In our present work, we herein present an efficient regioselective synthesis of novel 4-heteroaryl-pyrazoles, which have not been reported hitherto in a multicomponent synthesis under microwave irradiation. The structures of the newly synthesized compounds were established on the basis of spectroscopic evidences and their synthesis by alternative methods. The in vitro growth inhibitory activity of the synthesized compounds against hepatocellular carcinoma (HepG-2) and human lung cancer (A-549) cell lines were investigated in comparison with Cisplatin reference drug as a standard drug using MTT assay and the results revealed promising activities of six compounds.

\section{Abbreviations}

A-549: human lung cancer; HepG2: human hepatocellular carcinoma; EtOH: ethanol; mp: melting point; TEA: triethylamine; IR: infra-red; ATCC: American type culture collection; TLC: thin layer chromatography.

\section{Authors' contributions}

SMG designed research; SMG, ZAM, RAMF and MME performed research and analyzed the data. All authors read and approved the final manuscript.

\section{Author details}

${ }^{1}$ Department of Chemistry, Faculty of Science, Cairo University, Giza 12613, Egypt. ${ }^{2}$ Department of Organic Chemistry, National Organization for Drug Control and Research (NODCAR), Giza 12311, Egypt. ${ }^{3}$ Faculty of Science, King Khalid University, Abha, Kingdom of Saudi Arabia. ${ }^{4}$ Department of Chemistry, College of Science, King Saud University, P. O. Box 2455, Riyadh 11451 , Kingdom of Saudi Arabia.

\section{Acknowledgements}

The authors extend their sincere appreciation to the Deanship of Scientific Research at the King Saud University for its funding this Prolific Research group (PRG-1437-29). 


\section{Competing interests}

The authors declare that they have no competing interests.

\section{Sample availability}

Samples of the compounds are available from the authors.

\section{Publisher's Note}

Springer Nature remains neutral with regard to jurisdictional claims in published maps and institutional affiliations.

Received: 16 March 2017 Accepted: 2 May 2017

Published online: 08 May 2017

\section{References}

1. Murata T, Murai M, Ikeda Y, Miki K, Ohe K (2012) Pd- and Cu-catalyzed one-pot multicomponent synthesis of hetero alpha, alpha'-dimers of heterocycles. Org Lett 14:2296-2299

2. Lalli C, Bouma MJ, Bonne D, Masson G, Zhu J (2011) Exploiting the divergent reactivity of alpha-isocyanoacetate: multicomponent synthesis of 5-alkoxyoxazoles and related heterocycles. Chemistry 17:880-889

3. Zhang S, Zhang WX, Xi Z (2010) Efficient one-pot synthesis of N-containing heterocycles by multicomponent coupling of silicon-tethered diynes, nitriles, and isocyanides through intramolecular cyclization of iminoacylZr intermediates. Chemistry. 16:8419-8426

4. Lopez C, Zougagh M, Algarra M, Rodriguez-Castellon E, Campos BB, Esteves da Silva JC, Jimenez-Jimenez J, Rios A (2015) Microwave-assisted synthesis of carbon dots and its potential as analysis of four heterocyclic aromatic amines. Talanta 132:845-850

5. Agudelo Mesa LB, Padro JM, Reta M (2013) Analysis of non-polar heterocyclic aromatic amines in beefburguers by using microwave-assisted extraction and dispersive liquid-ionic liquid microextraction. Food Chem 141:1694-1701

6. Pawelczyk A, Zaprutko L (2009) Microwave assisted synthesis of unsaturated jasmone heterocyclic analogues as new fragrant substances. Eur J Med Chem 44:3032-3039

7. Jeselnik M, Varma RS, Polanc S, Kocevar M (2001) Catalyst-free reactions under solvent-free conditions: microwave-assisted synthesis of heterocyclic hydrazones below the melting points of neat reactants. Chemical Commun 18:1716-1717

8. Al-Qalaf F, Mandani F, Abdelkhalik MM, Bassam AA (2009) Synthesis of 5-substituted 3-amino-1H-pyrazole-4-carbonitriles as precursors for microwave assisted regiospecific syntheses of pyrazolo[1,5-a]pyrimidines. Molecules 14:78-88

9. Ezer M, Yildirim LT, Bayro O, Verspohl EJ, Dundar OB (2012) Synthesis and antidiabetic activity of morpholinothiazolyl-2,4-thiazolidindione derivatives. J Enzyme Inhib Med Chem 27:419-427

10. Zhang K, Yan JF, Tang XM, Liu HP, Fan L, Zhou GM, Yang DC (2011) Synthesis of novel beta-aminoalcohols containing nabumetone moiety with potential antidiabetic activity. Yaoxue Xuebao 46:412-421

11. Dutt P, Sarkar AK (1993) Alterations in rat intestinal sucrase and alkaline phosphatase activities in alloxan induced experimental diabetes. Indian J Biochem Biophys 30:177-180

12. Tarach JS (1978) Electronmicroscopic cytochemical studies on lysosomes and peroxisomes of a rat cardiac muscle in the experimental alloxan diabetes. Acta Histochem 62:32-43

13. Ranatunge RR, Garvey DS, Janero DR, Letts LG, Martino AM, Murty MG, Richardson SK, Young DV, Zemetseva IS (2004) Synthesis and selective cyclooxygenase-2 (COX-2) inhibitory activity of a series of novel bicyclic pyrazoles. Bioorg Med Chem 12:1357-1366

14. Sakya SM, Cheng H, Lundy KM, De Mello, Shavnya A, Minich ML, Rast B, Dutra J, Li C, Rafka RJ, Koss DA, Li J, Jaynes BH, Ziegler CB, Mann DW, Petras CF, Seibel SB, Silvia AM, George DM, Hickman A, Haven ML, Lynch MP (2006) 5-Heteroatom-substituted pyrazoles as canine COX-2 inhibitors: part 2. Structureeactivity relationship studies of 5-alkylethers and 5-thioethers. Bioorg Med Chem Lett 16:1202-1206
15. Reddy GJ, Pallavi K, Reddy RS, Reddy KS (2005) Synthesis of 1,3-diaryl4-(pyridine-4-yl)pyrazoles as a new class of pyrazole based diaryl heterocycles. Indian J Chem 44:812-814

16. Sauzem PD, Sant'Anna GDS, Machado P, Duarte MMMF, Ferreira J, Mello CF, Beck P, Bonacorso HG, Zanatta N, Martins MAP, Rubin MA (2009) Effect of 5-trifluoromethyl-4,5-dihydro-1H-pyrazoles on chronic inflammatory pain model in rats. Eur J Pharmacol 61:91-100

17. Koca I, Ozgur A, Coskun KA, Tutar Y (2013) Synthesis and anticancer activity of acyl thioureas bearing pyrazole moiety. Bioorg Med Chem 21:3859-3865

18. Tyagarajan S, Chakravarty PK, Zhou B, Taylor B, Eid R, Fisher MH, Parsons WH, Wyvratt MJ, Lyons KA, Klatt T, Li X, Kumar S, Williams B, Felix J, Priest BT, Brochu RM, Warren V, Smith M, Garcia M, Kaczorowski GJ (2010) Discovery of a novel class of biphenyl pyrazole sodium channel blockers for treatment of neuropathic pain. Bioorg Med Chem Lett 20:7479-7482

19. Pattan SR, Rabara PA, Pattan JS, Bukitagar AA, Wakale VS, Musmade DS (2009) Synthesis and evaluation of some novel substituted 1,3,4-oxadiazole and pyrazole derivatives for antitubercular activity. Indian J Chem 48B:1453-1456

20. Shih SR, Chu TY, Reddy G, Tseng SN, Chen HL, Tang WF, Wu MS, Yeh JY, Chao YS, Hsu J, Hsieh HP, Horng JT (2010) Pyrazole compound BPR1P0034 with potent and selective anti-influenza virus activity. J Biomed Sci 17:13

21. Lo HY, Man CC, Fleck RW, Farrow NA, Ingraham RH, Kukulka A, Proudfoot JR, Betageri R, Kirrane T, Patel U, Sharma R, Hoermann MA, Kabcenell A, Lombaert SD (2010) Substituted pyrazoles as novel sEH antagonist: investigation of key binding interactions within the catalytic domain. Bioorg Med Chem Lett 26:6379-6383

22. Kasımogullari R, Bulbul M, Arslan BS, Gokçe B (2010) Synthesis, characterization and antiglaucoma activity of some novel pyrazole derivatives of 5-amino-1,3,4-thiadiazole-2-sulfonamide. Eur J Med Chem 45:4769-4773

23. Padmaja A, Rajasekhar C, Muralikrishna A, Padmavathi V (2011) Synthesis and antioxidant activity of oxazolyl/thiazolylsulfonylmethyl pyrazoles and isoxazoles. Eur J Med Chem 46:5034-5038

24. Xia Y, Dong ZW, Zhao BX, Ge X, Meng N, Shin DS, Miao JY (2007) Synthesis and structure activity relationships of novel 1-arylmethyl-3-aryl-1H-pyrazole-5-carbohydrazide derivatives as potential agents against A549 lung cancer cells. Bioorg Med Chem 15:6893-6899

25. Daidone G, Maggio B, Raffa D, Plescia S, Schillaci D, Maria V, Raimondi (2004) Synthesis and in vitro antileukemic activity of new 4-triazenopyrazole derivatives. Farmaco 59:413-417

26. Farghaly AR (2010) Synthesis of some new indole derivatives containing pyrazoles with potential antitumor activity. ARKIVOC 11:177-187

27. Soliman EA, El-Zahar MI, El-Masry AH, Kamel M, Gohar RS (2010) Synthesis and anticancer evaluation of novel tetrahydronaphthalen-6-ylthiazole heterocycles against human HePG2 and MCF7 cell lines. Der Pharma Chem. 2:507-521

28. El-Zahar MI, EL-Karim SA, Haiba ME, Khedr MA (2011) Synthesis, antitumor activity and molecular docking study of novel benzofuran-2-yl pyrazole pyrimidine derivatives. Acta Pol Pharm Drug Res 68:357-373

29. Tanitame A, Oyamada Y, Ofuji K, Terauchi H, Kawasaki M, Wachi M Yamagishi J (2005) Synthesis and antibacterial activity of a novel series of DNA gyrase inhibitors: 5-[(E)-2-arylvinyl]pyrazoles. Bioorg Med Chem Lett 15:4299-4303

30. Dawood KM, Eldebss TMA, El-Zahabi HSA, Yousef MH, Metz P (2013) Synthesis of some new pyrazole-based 1,3-thiazoles and 1,3,4-thiadiazoles as anticancer agents. Eur J Med Chem 70:740-749

31. Gomha SM, Khali KD, El-Zanate AM, Riyadh SM (2015) A Facile Green Synthesis and anti-cancer activity of bis-arylhydrazononitriles, triazolo[5,1-c] [1,2,4]triazine, and 1,3,4-thiadiazoline. Heterocycles 87(5):1109-1120

32. Gomha SM, Badrey MG, Edrees MM (2016) Heterocyclization of a bis-thiosemicarbazone of 2,5-diacetyl-3,4-disubstituted-thieno[2,3-b]thiophene bis-thiosemicarbazones leading to bis-thiazoles and bis-1,3,4-thiadiazoles as anti-breast cancer agents. J Chem Res 40:120-125

33. Gomha SM, Kheder NA, AAbdelhamid AO, Mabkhot YN (2016) One pot single step synthesis and biological evaluation of some novel bis(1,3,4-thiadiazole) derivatives as potential cytotoxic agents. Molecules 21:15321-15328 
34. Gomha SM, Ahmed SA, Abdelhamid AO (2015) Synthesis and cytotoxicity evaluation of some novel thiazoles, thiadiazoles, and pyrido[2,3-d] $[1,2,4]$ triazolo[4,3-a]pyrimidin-5(1H)-one incorporating triazole moiety. Molecules 20:1357-1376

35. Gomha SM, Khalil KD (2012) A convenient ultrasound-promoted synthesis and cytotoxic activity of some new thiazole derivatives bearing a coumarin nucleus. Molecules 17:9335-9347

36. Gomha SM, Riyadh SM, Mahmmoud EA, Elaasser MM (2015) Synthesis and anticancer activity of arylazothiazoles and 1,3,4-thiadiazoles using chitosan-grafted-poly(4-vinylpyridine) as a novel copolymer basic catalyst. Chem Heterocycl Compd 51:1030-1038

37. Gomha SM, Riyadh SM, Abbas IM, Bauomi MA (2013) Synthetic utility of ethylidenethio-semicarbazide: synthesis and and anti-cancer activity of 1,3-thiazines and thiazoles with imidazole moiety. Heterocycles 87:341-356

38. Gomha SM, Abdel-aziz HM (2015) Synthesis and antitumor activity of 1,3,4-thiadiazole derivatives bearing coumarine ring. Heterocycles 91:583-592

39. Abbas IM, Gomha SM, Elaasser MM, Bauomi MA (2015) Synthesis and biological evaluation of new pyridines containing imidazole moiety as antimicrobial and anticancer agents. Turk J Chem 39:334-346

40. Gomha SM, Salah TA, Abdelhamid AO (2015) Synthesis, characterization and pharmacological evaluation of some novel thiadiazoles and thiazoles incorporating pyrazole moiety as potent anticancer agents. Monatsh Chem 146:149-158

41. Hassaneen HM, Mousa AH, Abeed NM, Shawali AS (1988) Chemistry of C-Heteroarylhydrazidoyl Halides. Synthesis and reactions of $\mathrm{N}$-( $\mathrm{p}$ Nitrophenyl)-C-(2-thienyl)-formohydrazidoyl Halides. Heterocycles 27:695-706
42. Al-Bogam AS, Mekky AEM (2016) Microwave-assisted regioselective synthesis of novel bis (azoles) and bis(azoloazines). J Heterocycl Chem 53:1554-1562

43. Zaki YH, Sayed AR, Elroby SA (2016) Regioselectivity of 1,3-dipolar cycloadditions and antimicrobial activity of isoxazoline, pyrrolo[3,4-d] isoxazole-4,6-diones, pyrazolo[3,4-d]pyridazines and pyrazolo[1,5-a] pyrimidines. Chem Central J. 10:17(1-13)

44. Eldebss TMA, Gomha SM, Abdulla MM, Arafa RK (2015) Novel pyrrole derivatives as selective $\mathrm{CHK} 1$ inhibitors: design, regioselective synthesis and molecular modeling. Med Chem Commun 6:852-859

45. Gaber HM, Bagley MC, Muhammad ZA, Gomha SM (2017) Recent developments in chemical reactivity of $\mathrm{N}, \mathrm{N}$-dimethylenamino ketones as synthons for various heterocycles. RSC Adv 7:14562-14610

46. Gomha SM, Shawali AS, Abdelhamid AO (2014) Convenient method for synthesis of various fused heterocycles via utility of 4-acetyl-5-methyl1-phenyl-pyrazole as precursor. Turk J Chem 38:865-879

47. Gomha SM, Abdelrazek FM, Abdelrahman AH, Metz P (2016) Synthesis of some novel thiazole, thiadiazole and 1,4-phenylene-bis-thiazole derivatives as potent antitumor agents. Heterocycles 92:954-967

48. Shawali S, Gomha SM (2000) A new entry for short and regioselective synthesis of $[1,2,4]$ triazolo[4,3-b][1,2,4]-triazin-7(1H)-one. Adv Synth Catal 342:599-604

49. Gomha SM, Riyadh SM, Mahmmoud EA, Elasser MM (2015) synthesis and anticancer activities of thiazoles, 1,3-thiazines, and thiazolidine using chitosan-grafted-poly(vinylpyridine) as basic catalyst. Heterocycles 91:1227-1243

\section{Submit your manuscript to a SpringerOpen ${ }^{\circ}$ journal and benefit from:}

- Convenient online submission

- Rigorous peer review

- Immediate publication on acceptance

- Open access: articles freely available online

- High visibility within the field

- Retaining the copyright to your article

Submit your next manuscript at $\boldsymbol{\nabla}$ springeropen.com 